\title{
On a class of discontinuous dynamical systems
}

\author{
Marius-Florin Danca
}




\title{
ON A CLASS OF DISCONTINUOUS DYNAMICAL SYSTEMS
}

\author{
MARIUS-FLORIN DANCA \\ Department of Mathematics, Spiru Haret College, \\ 3400 Cluj-Napoca, Romania \\ Marius.Danca@aut.utcluj.ro
}

[Received April 18, 2001]

\begin{abstract}
The aim of this paper is to find the conditions under which the discontinuous initial value problem $\dot{x}(t)=g(x(t))+\sum_{i=1}^{n} \alpha_{i} \operatorname{sgn} x_{i}(t) e^{i}, x(0)=x_{0}, t \in I=$ $[0, \infty)$, where $x_{0} \in \mathbb{R}^{n}, \quad \alpha_{i} \in \mathbb{R}$ and $e^{i}$ denotes the $i$-th canonical unit vector in $\mathbb{R}^{n}$, defines a dynamical system. A definition of dynamical systems which involves the existence and the uniqueness of solutions to initial value problems is presented. Because the problem may not have classical solutions, the Filippov regularization is used in order to restart the problem as a differential inclusion which may enjoy the existence and even uniqueness of generalized solutions. An illustrative example of this class of dynamical systems, a generalization of the Chua's circuit, is presented.
\end{abstract}

Mathematical Subject Classification: 49K24, 37M99

Keywords: Discontinuous initial value problems, dynamical systems, differential inclusions, set-valued maps.

\section{Introduction}

The paper is devoted to differential equations with discontinuous right-hand sides which model a whole variety of applications: dry friction, electrical circuits, oscillations in visco-elasticity, brake processes with locking phase, oscillating systems with viscous damping, elasto-plasticity, electrical circuits, forced vibrations, convex optimization, control synthesis of uncertain systems, etc. (see e.g. [1]-[5] and the references in [6], [7]). A large number of problems which belong to these applications can be described by the following autonomous initial value problem (i.v.p.)

$$
\dot{x}(t)=f(x(t)), \quad x(0)=x_{0}, \quad x_{0} \in \mathbb{R}^{n}, \quad t \in I=[0, \infty),
$$

with the right-hand side $f: \mathbb{R}^{n} \rightarrow \mathbb{R}^{n}$ a vector single-valued map, discontinuous with respect to the state variable $x$ and locally bounded in the metric space $\mathbb{R}^{n}$ (i.e., bounded on each bounded subset of $\mathbb{R}^{n}$ ). Our goal is to emphasize a class of i.v.p. (1.1) which define dynamical systems (d.s.). Throughout this paper the discontinuity is considered only with respect to the state variable $x$. Many authors consider the concept of continuity of a d.s. as being with respect to time variable, while others consider the continuity (or Lipschitz continuity) with respect to initial data in order to define a continuous d.s. (see e.g. [8], [9] and the references therein). However, 
in the case of continuity with respect to the state variable, for most of the standard assumptions leading to the existence and uniqueness of the solutions, the continuous dependence on initial data follows (see [9]).

There are many mathematical definitions for a d.s. (see e.g. [8], [9] and the references therein). Our definition uses the existence and, optionally, the uniqueness of the solutions to the i.v.p. (1.1). If the right-hand side of (1.1) is discontinuous with respect to $x$, the i.v.p. need not have classical solutions and another concept of solution must be used. One of the typical cases for discontinuous i.v.p. is modeled by the so-called switch problems.

Example 1.1. [10] Consider the discontinuous right-hand side equation $\dot{x}=1-$ $2 \operatorname{sgn} x$, with the classical solutions

$$
x(t)=\left\{\begin{array}{cc}
3 t+C_{1}, & x<0 \\
-t+C_{2}, & x>0
\end{array} \quad, \quad C_{1}, C_{2} \in \mathbb{R} .\right.
$$

As $t$ increases, the classical solutions tend to the line $x=0$, but it cannot be continued along this line, since the map $x(t)=0$ so obtained does not satisfy the equation in the usual sense (for it, $\dot{x}(t)=0$ and the right-hand side has the value $1-2 \operatorname{sgn} 0=1$ ). Hence there are no classical solutions of i.v.p. starting with $x(0)=0$. Therefore a generalization of the concept of solution is required.

To get around to a solution of the i.v.p. (1.1) with a discontinuous right-hand side, the problem may be restarted as a differential inclusion (d.i.)

$$
\dot{x}(t) \in F(x(t)), \quad x(0)=x_{0}, \quad \text { for a.a. } t \in I,
$$

where $\quad F: \mathbb{R}^{n} \rightrightarrows \mathbb{R}^{n}$ is a vector set-valued map into the set of all subsets of $\mathbb{R}^{n}$ which can be defined in several ways. For the background and a comprehensive theory of d.i. and set-valued maps we refer the reader to [10]-[12].

The simplest convex definition of $F$ is obtained by the so-called Filippov regularization [10]

$$
F(x)=\bigcap_{\varepsilon>0} \overline{\operatorname{conv}\left(f\left(\left\{z \in \mathbb{R}^{n}:\|z-x\| \leq \varepsilon\right\} \backslash M\right)\right)},
$$

where $F(x)$ is the convex hull of $f, \mu$ denotes the Lebesgue measure in $\mathbf{R}^{n}$, conv is the convex hull, $M$ is a null set and $\varepsilon$ the radius of the ball centered in $x$. In the points where the map $f$ is continuous, $F(x)$ consists of one point which coincides with the value of $f$ at this point (i.e., we get back $f(x)$ as the right-hand side). In the points belonging to $M$, the set is given by (1.3). Details and other regularization procedures can be found in [10].

In order to justify the use of d.i. (1.2-1.3) to a physical system, we must use a small $\varepsilon$ so that the motion of the physical system is arbitrarily close to a certain solution of d.i. (for instance, it tends to the solution as $\varepsilon \rightarrow 0$ ). 
As an example, the Filippov regularization of the usual sign map is the signum set-valued map

$$
\operatorname{Sgn} x=\left\{\begin{array}{ll}
\{-1\}, & x<0 \\
{[-1,1],} & x=0 \\
\{+1\}, & x>0
\end{array} .\right.
$$

Remark 1.2. Embedding $f$ into a set-valued map $F$, which has enough regularity, closely related to the trajectories of the original differential equation, we can stress the point that whenever $f$ is continuous at $x$, then a solution to d.i. (1.2) satisfies the i.v.p. (1.1). Certainly, any classical solution to the i.v.p. (1.1) is a solution to the i.v.p. (1.2). Hence, as stipulated in [10], we are justified to call a solution of i.v.p. (1.1) a solution of the i.v.p. (1.2).

On mild assumptions, d.i. (1.2) has generalized (Filippov) solutions that happen to be even a.e. unique, but it could have multiple solutions as well.

Example 1.3. Let the discontinuous i.v.p. $\dot{x}=\operatorname{sgn} x, x(0)=0$. There is no classical solution starting from 0 . However, considering the corresponding d.i. $\dot{x} \in$ $F(x)=\operatorname{Sgn} x$, there are multiple Filippov solutions: $x(t)=0$ for $t \leq t_{*}$ and $x(t)=$ $\pm\left(t-t_{*}\right)$ for $t>t_{*}$, where $t_{*} \geq 0$ could be $\infty$.

Example 1.4. If we consider the i.v.p. $\dot{x} \in-\operatorname{Sgn} x, x(0)=0$, then there is a unique Filippov solution

$$
x(t)=\left\{\begin{array}{ll}
t_{*}-t, & t \leq t_{*} \\
0, & t>t_{*}
\end{array},\right.
$$

and the trajectory can be continuously extended from $x=0$ for $t>t_{*}$.

The main objective is the study of conditions under which the discontinuous i.v.p. modeling the switch systems

$$
\dot{x}(t)=g(x(t))+\sum_{i=1}^{n} \alpha_{i} \operatorname{sgn} x_{i}(t) e^{i}, \quad x(0)=x_{0}, \quad t \in I=[0, \infty),
$$

with $g: \mathbb{R}^{n} \rightarrow \mathbb{R}^{n}$ a vector single-valued map, $\alpha_{i} \in \mathbb{R}$ and $e^{i}$ the $i$-th canonical unit vector in $\mathbb{R}^{n}$ could be viewed as a switch d.s. Consequently, we introduce in Section 2 a definition for switch d.s. considering the existence and optionally the uniqueness of solution to i.v.p. (1.4). The existence (Péano's theorem for d.i.) and uniqueness (special Lipschitz conditions) of solutions of i.v.p. (1.4) will be analyzed through the corresponding d.i. obtained by Filippov regularization. The explicit Euler method for d.i. necessary to numerical dynamics is also presented. The main result of this paper is introduced in Section 3 and states the assumptions in which the i.v.p. (1.4) defines a switch d.s.

\section{Preliminaries}

Definition 2.1. A (Filippov) solution of i.v.p. (1.2) is an absolutely continuous vector-valued map $x: I \rightarrow \mathbb{R}^{n}$ satisfying (1.2), for a.a. $t$ on $I$. 
See [10] and [13] for properties of Filippov solutions.

Definition 2.2. The i.v.p. (1.1) is said to define a generalized switch d.s. on $\mathbb{R}^{n}$ if for every $x_{0}$ there exists a solution of i.v.p. (1.1) defined for a.a. $t \in I$. If the solution is a.e. unique, then the i.v.p. is said to define a switch d.s.

To establish conditions in which a d.i. admits solutions, few basic properties of the set-valued maps are presented next.

Let $X$ and $Y$ be two non-empty sets. For practical reasons it is convenient to characterize a vector set-valued map $F: X \rightrightarrows Y$ by its graph

$$
\operatorname{Graph}(F):=\{(x, y) \in X \times Y \mid y \in F(x)\} .
$$

Remark 2.3. Due to the symmetric interpretation of a set-valued map as a graph (see e.g. [12]), we shall say that a set-valued map satisfies a property if and only if its graph satisfies it. For instance a set-valued map is said to be closed if and only if its graph is closed.

Notation 2.4. Let denote with $\mathcal{P}$ the class of the so-called Péano maps i.e. upper semicontinuous (u.s.c.) maps with non-empty closed and convex values, called basic or Péano conditions.

Definition 2.5. [11], [12] A single-valued map $f: \mathbb{R}^{n} \rightarrow \mathbb{R}^{n}$ is called a selection of the set-valued $F$ map if

$$
\forall x \in \mathbb{R}^{n}, \quad f(x) \in F(x)
$$

Generally, a set-valued map $F$ admits (infinitely) many selections, each of them giving rise to a differential equation $\dot{x}(t)=f(x(t))$ and whose classical solutions (if any) are Filippov solutions of (1.2). There are many ways to choose a selection (see [6]). The selections could be discontinuous (both with respect $t$ and $x$ ) . Let us consider as an example the set-valued map $F: X \rightrightarrows Y$ with closed convex images, $X$ being a metric space and $Y$ a Hilbert space. The single-valued map

$$
m(F(x)):=\left\{u \in F(x) \mid\|u\|=\min _{y \in F(x)}\|y\|\right\},
$$

is called the minimal norm selection. For the sign set-valued map, the minimal selection is the discontinuous single-valued map

$$
m(\operatorname{Sgn} x)=\left\{\begin{array}{ll}
-1, & x<0 \\
0, & x=0 \\
+1, & x>0
\end{array} .\right.
$$

An important condition to extend the existence interval for a solution of the i.v.p. $(1.2)$ to $I=[0, \infty)$ is the compactness of the minimal selection.

Notation 2.6. We say that condition $\mathcal{C}$ is satisfied if there exists a compact subset $C \subset \mathbb{R}^{n}$ such that $m(F(x)) \subseteq \mathcal{C}$ for all $x \in \mathbb{R}^{n}$. 
Definition 2.7. $F$ satisfies a growth condition (g.c.) on $\mathbb{R}^{n}$ if there exist constants $K_{1}, K_{2} \geq 0$ with

$$
\|\xi\| \leq K_{1}\|x\|+K_{2}
$$

for all $\xi \in F(x), x \in \mathbb{R}^{n}$.

The g.c. implies that all solutions and selections remain in some bounded subset and it is used instead of global boundedness of the right-hand side (compare [2], [7] and $[14])$.

Remark 2.8. i) For $f$ locally bounded, the set-valued map $F$ defined by (1.3) has the following properties: is u.s.c. with non-empty closed and convex values, i.e., a Péano map (compare [11, Proposition 1 p.102] and [10, Lemma 3, p.67]).

ii) Moreover, because the convex hull of a finite set is compact, $F(x)$ given by (1.3) is a compact set (see [11, Corollary 1, p.20]). Hence, it is easy to verify that $F$ verifies $\mathcal{C}$ condition.

iii) The Sgn set-valued maps satisfies the g.c.

In order to prove the uniqueness of solutions for certain class of d.i., special Lipschitz conditions are necessary.

Definition 2.9. $F$ satisfies a uniform one-sided Lipschitz condition (o.s.L.) with o.s.L. constant $\lambda$ if we have

$$
\left(\zeta^{\prime}-\zeta^{\prime \prime}, x^{\prime}-x^{\prime \prime}\right) \leq \lambda\left\|x^{\prime}-x^{\prime \prime}\right\|
$$

uniformly in $t$ and for all $\zeta^{\prime} \in F\left(x^{\prime}\right), \zeta^{\prime \prime} \in F\left(x^{\prime \prime}\right)$, with $x^{\prime}, x^{\prime \prime} \in \mathbb{R}^{n}$.

The o.s.L. is weaker than the classical Lipschitz condition or Lipschitz continuity. For higher dimensional problems and explicit numerical methods, the usual o.s.L. is no longer adequate to prove uniqueness of solution. Hence we need a stronger version, the strengthened one-sided Lipschitz condition introduced in [15] (see also [16] and $[17])$.

Definition 2.10. [15] $F$ satisfies a strengthened one-sided Lipschitz condition (s.o.s.L.) with o.s.L. constants $\left(\lambda_{1}, \lambda_{2}, \ldots, \lambda_{n}\right)$ if the implication

$$
x_{i}^{\prime}>x_{i}^{\prime \prime} \Rightarrow \zeta_{i}^{\prime} \leq \zeta_{i}^{\prime \prime}+\lambda_{i}\left\|x^{\prime}-x^{\prime \prime}\right\|,
$$

is true for all and all $x^{\prime}, x^{\prime \prime} \in \mathbb{R}^{n}, \zeta_{i}^{\prime} \in F\left(x^{\prime}\right), \zeta_{i}^{\prime \prime} \in F\left(x^{\prime \prime}\right)$ and all components $i=1,2, \ldots, n$.

Remark 2.11. i) For $n>1$ the s.o.s.L. is stronger than o.s.L. and weaker than the classical Lipschitz condition for single-valued right-hand sides and, if $n=1$, the s.o.s.L. and o.s.L. are equivalent (see [18]).

ii) The unidimensional set-valued map $-\operatorname{Sgn} x$ satisfies the s.o.s.L., while $+\operatorname{Sgn} x$ does not. The o.s.L./ s.o.s.L. conditions are only sufficient conditions for uniqueness. Thus a general criterion for uniqueness does not exist. However, for our class of problems, the positiveness of some $\alpha_{k}$ in (1.4) seems to be adequate for nonuniqueness (see Examples 1.2 and 1.3). 
In [18] the following lemma is proved

Lemma 2.12. [18] Let the set-valued map $F$ be decomposable in the following form

$$
F(x)=g(x)+\sum_{i=1}^{n} \beta_{i}(x) e^{i},
$$

for all $x \in \mathbb{R}^{n}$, where the single-valued map $g: \mathbb{R}^{n} \rightarrow \mathbb{R}^{n}$ is Lipschitz continuous, the set-valued maps $\beta_{i}: \mathbb{R} \rightarrow \mathbb{R}$ satisfy o.s.L. and $e^{i}$ denotes the $i$-th canonical unit vector in $\mathbb{R}^{n}$. Then $F$ satisfies s.o.s.L.

Let $N$ be a natural number $N \in \mathbf{N}^{\prime} \subset \mathbb{N}, \mathbf{N}^{\prime}$ denoting a subsequence of $\mathbb{N}$ tending to infinity, $T>0, h=T / N$, and an equidistant grid

$$
t_{0}<t_{1}<\ldots<t_{N}=T .
$$

We associate with (1.2) a sequence of discrete-time inclusions in the form

$$
y_{k+1} \in G_{k}^{N}\left(h ; y_{k}\right), \quad k=0,1, \ldots, N-1, y_{0}=x_{0},
$$

where $G_{k}^{N}: \mathbb{R}^{n} \rightrightarrows \mathbb{R}^{n}$ is a discrete-time set-valued map. A solution of (2.2) on $[0, T]$ is any sequence of $N+1$ vectors $y_{0}, y_{1}, \ldots, y_{N}$ that satisfies $(2.2)$ for $k=$ $0,1, \ldots, N-1$. The simplest explicit difference method for d.i. is the set-valued version of the classical Euler discretization method for differential equation with

$$
G_{k}^{N}\left(h ; y_{k}\right)=y_{k}+h F\left(t_{k}, y_{k}\right) .
$$

The Euler convergence theorem is presented in many works, and various forms (see e.g. [10 Theorem 1, p.77], [11, Theorem 3, p.98], [12, Theorem 10.1.3, p.390], or the papers [7], [14], [15], [18-20]), and uses the idea of the classical Péano theorem to prove the existence of solutions to d.i. Selections strategies for (2.2) can be found in [21].

The existence and uniqueness of solutions to a d.i. were treated in many ways and regard the convergence of difference methods to solve a d.i. The following theorem is the existence Péano's Theorem to d.i.

Theorem 2.13. (Péano's Theorem, [11]) Let the i.v.p. (1.2) verify the following assumptions: $F \in \mathcal{P}$ and verify the condition $\mathcal{C}$. Then there exists a solution of i.v.p. $(1.2)$ on $[0, \infty)$.

The proof can be found e.g. in [11, Theorem 4 p.101] or [20].

Remark 2.14. In practical problems, it is more convenient to consider the closure of the graph of $F$ instead the closed values of $F$ (see Remark (2.3) and [18] where a practical variant of Theorem 2.13 using g.c. is presented).

Using the strengthened version of o.s.L., we can prove the following corollary ensuring the uniqueness of the solutions of i.v.p. (1.2). 
Corollary 2.15. For the i.v.p. (1.2) with F satisfying a s.o.s.L. there exists at most one solution.

Proof. Because $F$ satisfies the s.o.s.L, it satisfies the o.s.L. as well (see Remark 2.11i) and in [19] uniqueness of differential equations with right-hand sides satisfying the o.s.L. condition is proved (the subject is also treated in [10, Theorem 2, Remark 11, p.5 and Theorem 1 p.106]).

Remark 2.16. By uniqueness of a solution we mean here that a solution lying on a surface of discontinuity of the right-hand side or on an intersection of surfaces of discontinuities of i.v.p. (1.1) can be uniquely continued in the positive direction of time. Note that uniqueness in the positive time direction does not necessarily imply the uniqueness in the negative time.

\section{Switch dynamical systems}

Let us consider now our class of switched i.v.p. (1.4). Using the Filippov regularization, the corresponding d.i. is

$$
\dot{x}(t) \in F(x(t))=g(x(t))+\sum_{i=1}^{n} \alpha_{i} \operatorname{Sgn} x_{i} e^{i}, x(0)=x_{0}, \text { for a.e. } t \in I .
$$

Then the main result of this paper is the following theorem

Theorem 3.1. Let $g$ be Lipschitz continuous and satisfy a g.c. Then the i.v.p. (1.4) defines a generalized switch d.s. If, moreover, all $\alpha_{i}$ are negative, then the i.v.p. (1.4) defines a switch d.s.

Proof. Using Remark 2.8 iii) the right-hand side of (3.1) verifies a g.c. Hence it is locally bounded and the Filippov regularization can be used. The set-valued map $F$ so obtained, belongs to $\mathcal{P}$. Thus the i.v.p. (1.4) has solutions (Theorem 2.13). The maximal interval of existence is $I=[0, \infty)$ because $F$ verifies $\mathcal{C}$ condition (see Remark $2.8 \mathrm{ii}$ ). Thus the i.v.p. defines a generalized switch d.s. If all coefficients $\alpha$ are negative, then $\alpha_{i} \operatorname{Sgn} x_{i}$ are set-valued maps verifying o.s.L. (see Remark $2.11 \mathrm{ii}$ ) and using Lemma 2.12, $F$ verifies s.o.s.L. Hence the solution is unique (see Corollary 2.15) and the i.v.p. (1.4) defines a switch d.s.

Application: Let us consider the following discontinuous right-hand side differential equations modeling a Chua circuit [22]

$$
\begin{aligned}
& \dot{x_{1}}=-\alpha(b+1)\left(x_{1}-k \operatorname{sgn} x_{1}\right)+\alpha x_{2} \\
& \dot{x_{2}}=x_{1}-x_{2}+x_{3} \\
& \dot{x_{3}}=-\beta x_{2}
\end{aligned}
$$


which has no global classical solutions in the classical sense on $[0, \infty)$. Using the parameters value indicated in $[20]:-\alpha(b+1)=-2.57, \alpha=9, \beta=15.7$ and $k=1.5$, the behavior becomes chaotic (see [8] for details about the chaotic behavior of a d.s.). The Filippov regularization gives us the following d.i.

$$
\dot{x}(t) \in F(x(t)) \text { with } F(x(t))=\left(\begin{array}{l}
-2.57 x_{1}+9 x_{2} \\
x_{1}-x_{2}+x_{3} \\
-\beta x_{2}
\end{array}\right)+3.86 \operatorname{Sgn} x_{1} e^{1}
$$

The discontinuity surface, $S$, is given by the equation $x_{1}=0$. If we denote $f=$ $\left(f_{1}, f_{2}, f_{3}\right)^{T}, F=\left(F_{1}, F_{2}, F_{3}\right)^{T}$, then the graph of the set-valued map $F_{1}$ at the discontinuity points $\left(0, x_{2}, x_{3}\right), F_{1}\left(0, x_{2}\right)=9 x_{2}+[-3.86,3.86]$, is the dashed shape in Figure 1. The assumptions in Theorem 3.1 are verified and the i.v.p. (3.2) defines a generalized switch d.s., the solutions of d.i. (3.3) not being unique (see Remark 2.11ii). The solutions, chaotic for $\beta=15.7$, pass from one side of $S$ to the other (see Figure 2). To obtain the plot, a Matlab model was created (Figure 3). The chaotic behavior for this value of the parameter $\beta$ can be deduced for the bifurcation diagram as well (see Figure 4, where the maxima of $x_{3}$ versus $\beta$ was plotted), and also from the Poincaré section with the plane $x_{2}=0$ (Figure 5). Hence, for this value of $\beta$, the bifurcation diagram and Poincaré section indicate that there is no visible periodical motion. Details on bifurcations and Poincaré sections for continuous d.s. can be found in [8] and for discontinuous d.s. in [2]. In Figure 6 (a) and (b) phase portraits and time series of a trajectory are represented. The plots in Figures 4, 5, 6 were obtained with a Turbo Pascal code using the explicit Euler method.

Remark 3.2. i) This type of motion, when the solution passes through a point of the surface $S$ and goes off it, is called sliding motion [2].

ii) If we replace $+3.86 \operatorname{Sgn}_{1}$ in (3.2) with $-3.86 \operatorname{Sgn} x_{1}$, the solution becomes unique, i.e. (3.2) defines a switch d.s. Now the solution remains on $S$ for $t>$ $t_{*}$ (i.e., slides along the switching space; see [2] where details on these cases can be found), $t_{*}$ being the moment when the solution enters $S$. Next, the solution tends asymptotically to $(0,0,0)$ (Figure 7$)$. No chaotic behavior was found in this case. The oscillations on the planes $\left(x_{1}, x_{2}\right)$, and $\left(x_{1}, x_{3}\right)$, where the solution is in fact a Filippov solution, are typical of (especially explicit) numerical methods for differential inclusions. To avoid this situation, highly consistent methods must be used, e.g. Runge-Kutta method for differential inclusions (see e.g. [6] for details).

iii) The non-uniqueness of solutions could be interpreted here in the following manner: the value of $\dot{x}_{1}$ at zero value of the state variable $x_{1}$, for each $x_{2} \in[-0.43,+0.43]$, is uncertain and can take any value in the range $9 x_{2}+[-3.86,+3.86]$. Hence each trajectory of (3.2) can be considered to be a possible motion of the system. 


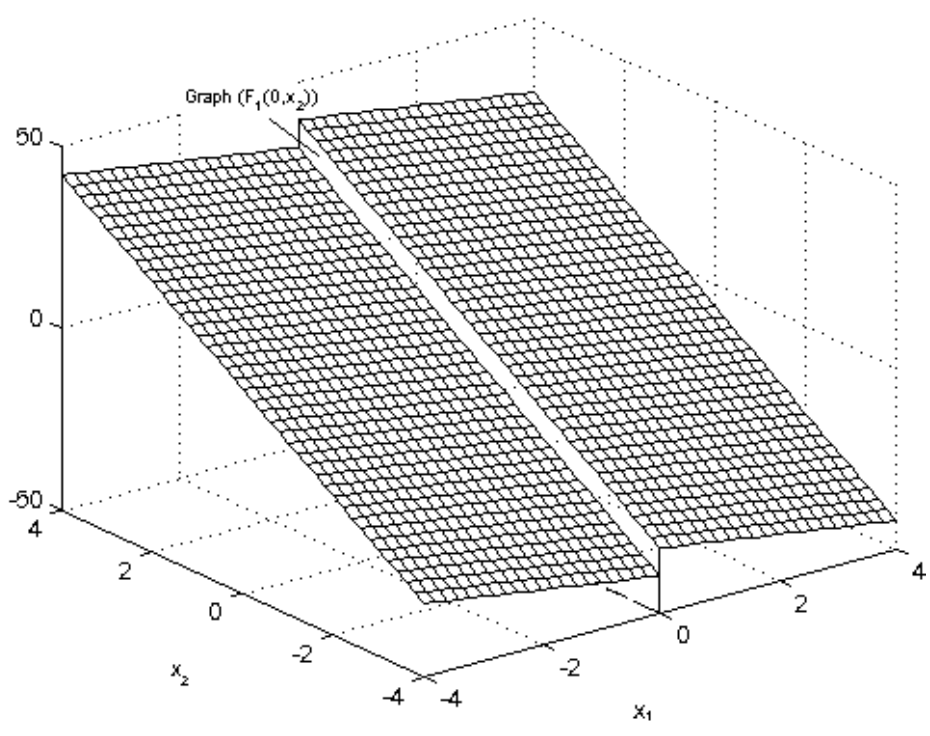

Figure 1 . The first component $F_{1}\left(0, x_{2}\right)$ of the set-valued map $F$ given by $(3.2)$

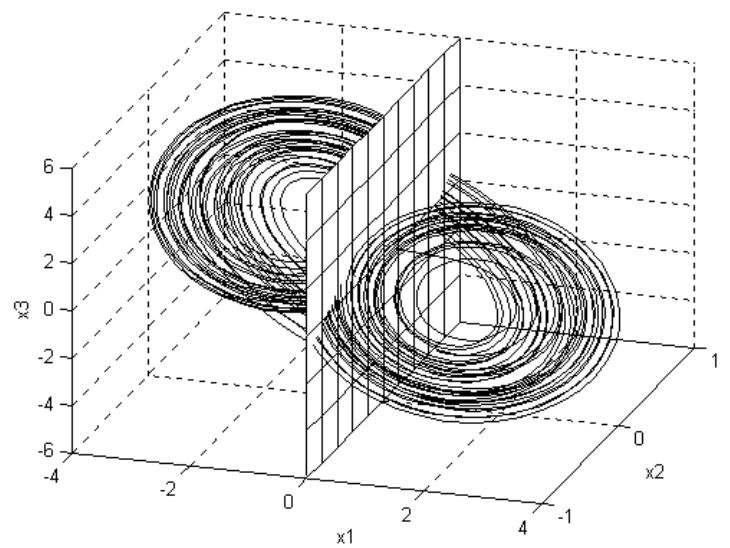

Figure 2. A chaotic trajectory and the discontinuity surface (Matlab) 


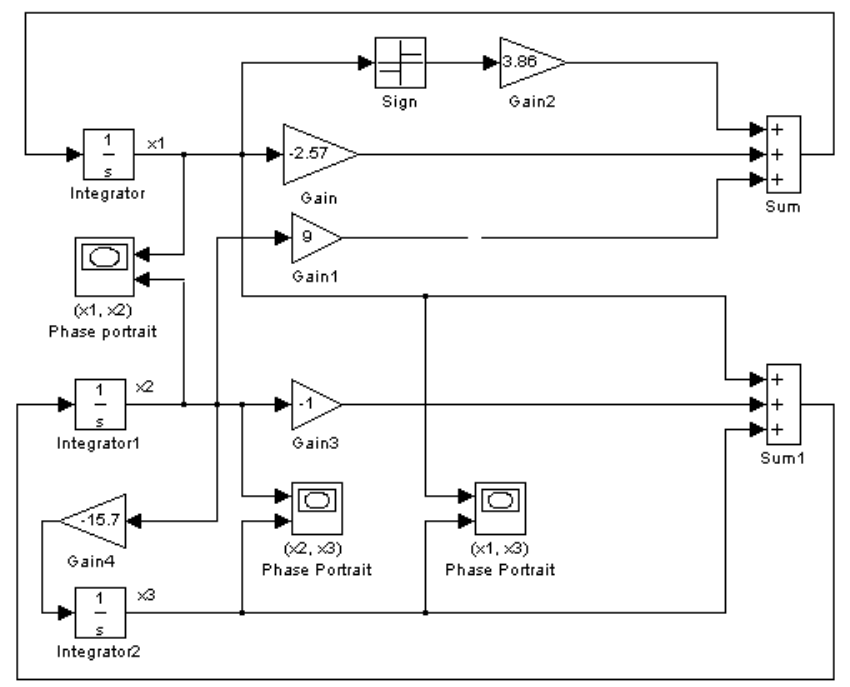

Figure 3. The block scheme of the system (3.2) (Simulink)

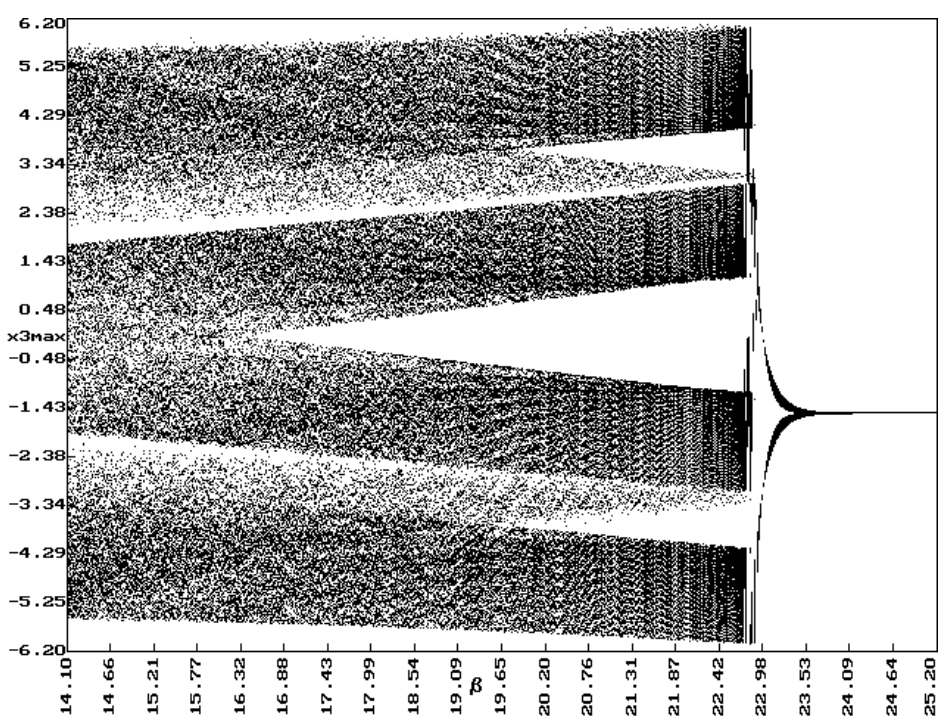

Figure 4. The bifurcation diagram for the generalized switch d.s. (3.2) for $\beta \in[14.10,25.20]$ 


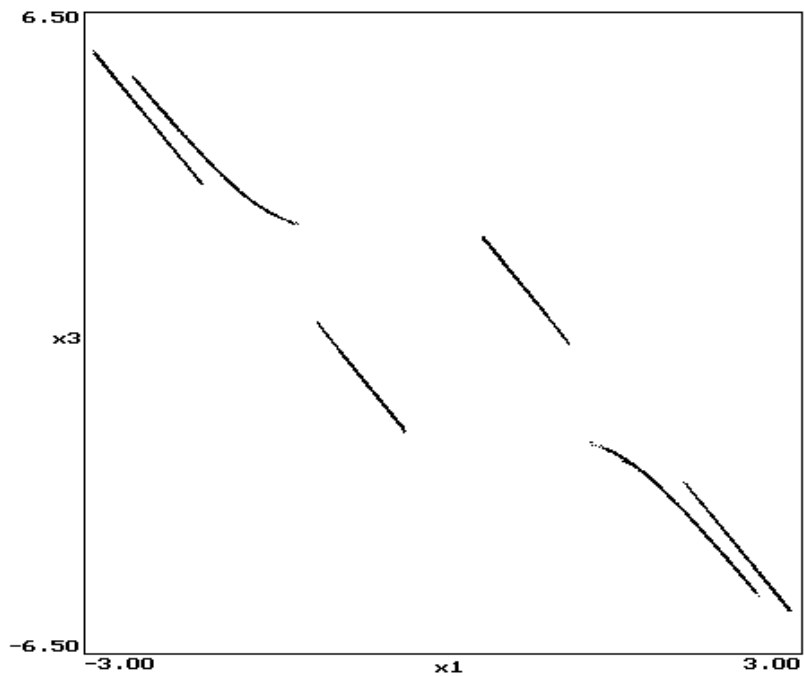

Figure 5. The Poincaré section of the system for $x_{2}=0$

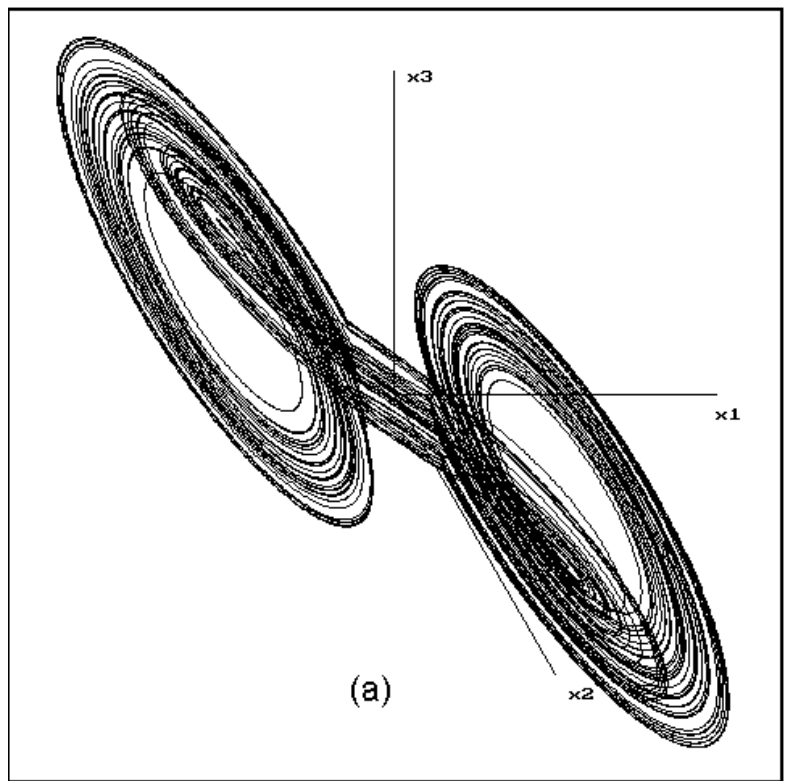

Figure 6(a). A chaotic trajectory for $\beta=15.7$ obtained with explicit Euler method with stepsize $h=0.005$ : a) Three-dimensional phase portrait view; 


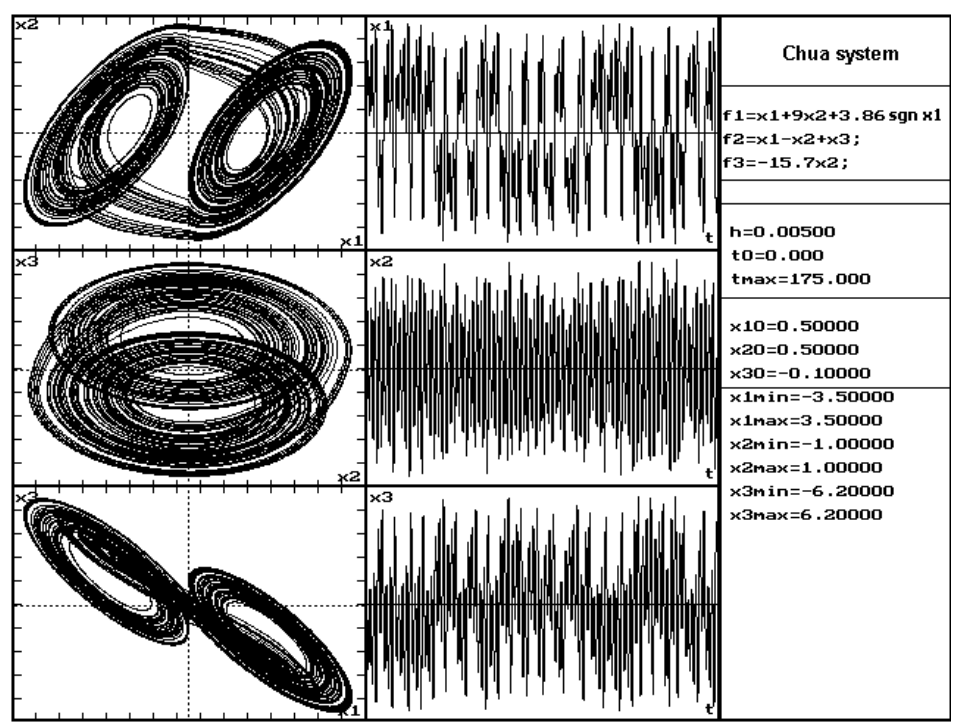

(b)

Figure 6(b). A chaotic trajectory for $\beta=15.7$ obtained with explicit Euler method with stepsize $h=0.005$ : b) Phase portraits and time series

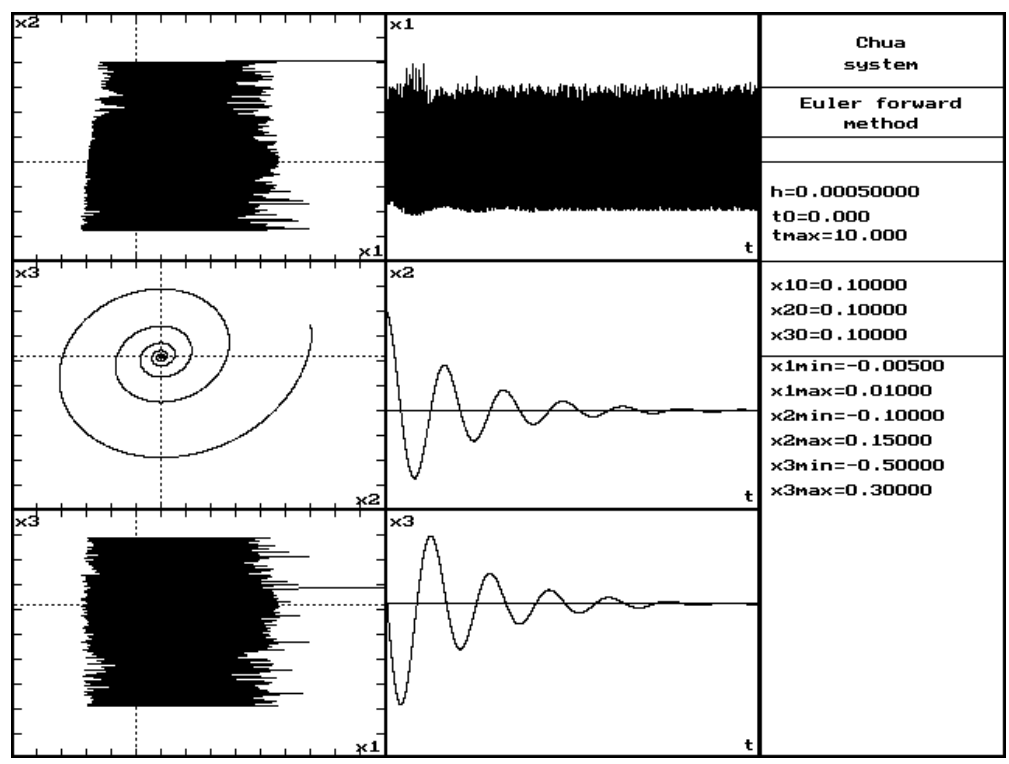

Figure 7. A trajectory of $(3.2)$ with $-3.86 \operatorname{Sgn}\left(x_{1}\right)$ instead $+3.86 \operatorname{Sgn}\left(x_{1}\right)$ and stepsize $h=0.0005$ 


\section{Conclusions}

In this paper we have focused on a class of discontinuous i.v.p. which can be viewed as defining switch d.s. Therefore a definition for a class of discontinuous dynamical systems, starting from a discontinuous i.v.p. was presented. Using well known results on the existence (as Péano's Theorem) and some criteria for uniqueness of solutions to d.i., we introduced a theorem which states the assumptions in which the i.v.p. could be viewed as a model of a (generalized) switch d.s.

The class of i.v.p. (1.4) was treated in [23] using the maximal monotonicity of Sgn $x$. In [24] the Approximate selection Theorem ([11], [12]) was used.

In [25] the synchronization of switch d.s. modeled by the i.v.p. (1.4) is treated and in [26] the explicit Euler method is considered as an d.s. which approximates the switch d.s. (1.4).

Acknowledgement: The author expresses his gratitude to Professor J. Kolumban, Professor N. Vornicescu and Professor D. Trif for their many helpful suggestions.

\section{REFERENCES}

[1] Deimling, K.: Multivalued Differential Equations, Volume 1 of de Gruyter series in Nonlinear Analysis and Applications, Walter de Gruyter, Berlin-New York, 1992.

[2] Wiercigroch, M. and DE Kraker, B.: Applied Nonlinear Dynamics and Chaos of Mechanical Systems with Discontinuities, World Scientific, Singapore, 2000.

[3] Popp, K. and Stelter, P.: Stick-slip vibrations and chaos, Philos. Trans. R. Soc. London, A323, (1990), 89-105.

[4] Buhite, J. L. and Owen, D. R.: An ordinary differential equation from the theory of plasticity, Arch. Rational Mech. Anal, 71, (1979), 357-383.

[5] Butenin, N. V., Nejmark, Y. I. and Fufaev, N. A.: An introduction to the Theory of Nonlinear Oscillations, Nauka, 1987. (in Russian)

[6] Dontchev, A. and Lempio, F.: Difference Methods for Differentiale Inclusions: a Survey, SIAM Review, 34(2), (1992), 263-294.

[7] Kaster-Maresch, A.: Implicit Runge-Kutta methods for differential inclusions, Numer. Funct. Anal. and Optimiz., 11, (1990-91), 937-958.

[8] Schuster, H. G.: Deterministic Chaos, An Introduction, VCH, Berlin, 1989.

[9] Stuart, A. M. and Humphries, A. R.: Dynamical Systems and Numerical Analysis, Cambridge University Press, 1996.

[10] Filippov, A. F.: Differential Equations with Discontinuous Right-hand Sides, Kluwer Academic Publishers, Dordrecht, 1988.

[11] Aubin, J.-P. and Cellina, A.: Set-valued Maps and Viability Theory, Springer-Verlag, Berlin, 1984.

[12] Aubin, J.-P. and Frankowska, H.: Set-Valued Analysis, Birkhäuser, 1990.

[13] HÁjek, O.: Discontinuous differential equations I, II, Diff. Equat., 32, (1979), 149-170.

[14] Taubert, K.: Converging Multistep Methods for Initial Value Problems Involving MultiValued Maps, Computing, 27, (1981), 123-136. 
[15] Lempio, F. : Modified Euler Methods for Differential Inclusions, in A. B. Kurzhanski, V. M. Veliov, Set-valued Analysis and Differential Inclusions. A Coll. of Papers resulting from a Workshop held in Pamporovo, Bulgaria, Sept. (1990).

[16] Lempio, F. and Veliov, V.: Discrete approximation of differential inclusions, GammMitteilungen, 2(21), (1998), 103-135.

[17] Lempio, F. and Veliov,V.: Discrete approximation of differential inclusions, Bayreuth Mathematische Schriften, 54, (1998), 149-232.

[18] Lempio, F.: Euler's method revisited, Proceedings of Steklov Institute of Mathematics Moscow, 211, (1995), 473-494.

[19] Filippov, A. F.: Differential equations with discontinuities right-hand side, AMS Transl., 42, (1964), 199-231.

[20] Lempio, F.: Difference Methods for Differential inclusions, Lecture Notes in Economics and Mathematics Systems, 378, (1992), 236-273.

[21] Kastner-Maresch, A. and Lempio, F.: Difference methods with selection strategies for differential inclusions, Numer. Funct. Anal. and Optimiz., 14, (1993), 555-572.

[22] Braun, R.: Generalizations of the Chua equations, IEEE Transactions on Circuits and Systems, Fundamental Theory and Applications, 40(11), (1993), 878-883.

[23] Elliott, C. M.: On the convergence of a one-step method for numerical solution of an ordinary differential inclusion, IMA Journal of Numerical Analysis, 5, (1985), 3-21.

[24] Danca, M.-F. and Codreanu, S.: On a possible approximation of discontinuous dynamical systems, Chaos, Solitons and Fractals, to be published.

[25] Danca, M.-F.: Synchronization of switch dynamical systems, Int. J. Bif. Chaos, to be published.

[26] Danca, M.-F.: The explicit Euler method for differential inclusions as approximation for switch dynamical systems, Journal of Nonlinear Science, submitted. 